\title{
Distal pancreatectomy with coeliac axis resection (Appleby procedure)
}

\author{
Prabath Kumarasinghe ${ }^{1}$, Ruwan Dissanayaka ${ }^{1}$, Buddika Uragoda ${ }^{2}$, Dulani Benaragama ${ }^{3}$, \\ Deepaka Weerasekara ${ }^{3}$ \\ ${ }^{1}$ National Hospital of Sri Lanka \\ ${ }^{2}$ Professorial unit, Colombo North Teaching Hospital \\ ${ }^{3}$ Faculty of Medical Sciences, University of Sri Jayewardenepura
}

Keywords: Coeliac axis; distal pancreatectomy; aorta; complications; liver

\section{Introduction}

Pancreatic neck and body cancer are frequently diagnosed at a locally advanced stage. According to current recommendations, involvement of superior mesenteric artery (SMA) or coeliac axis (CA) is no longer a contraindication for surgery [1]. In a selected subset of patients' coeliac axis resection with distal pancreatectomy (DP-CAR) can be done safely without vascular reconstruction.

\section{Case presentation}

A sixty-four-year-old woman presented with persistent epigastric pain with significant loss of appetite and loss of weight of $6 \mathrm{~kg}$ in 4 months duration. On further evaluation with MDCT pancreatic neck and body tumour was detected with tumour encasement ( $>180$ degrees) of the coeliac axis. She had no evidence of distant metastasis on cross-sectional imaging and CT mesenteric angiography confirmed patent pancreatoduodenal vascular arcade between a gastroduodenal artery (GDA) and SMA. She was planned for distal pancreatectomy with a coeliac axis resection (DP-CAR).

At the peritoneal survey, there were no distant metastases. There was a solid tumour involving the body and tail of the pancreas with the involvement of the coeliac axis $(>180$ degrees) and its branches. Common hepatic artery (CHA) was involved by the tumour up to the GDA junction. Clamp placed at $\mathrm{CHA}$ and liver doppler was done to confirm retrograde hepatic perfusion.

The pancreas was tunnelled anterior to the portal vein. SMA was explored and it was uninvolved. Pancreas transected at the neck away from the tumour preserving the GDA. CHA has transected $1 \mathrm{~mm}$ away from the GDA junction and closed the distal end with 5.0 prolene. The left gastric artery was divided close to the stomach. Further dissection was carried along the anterior surface of SMA to reach the anterior surface of the

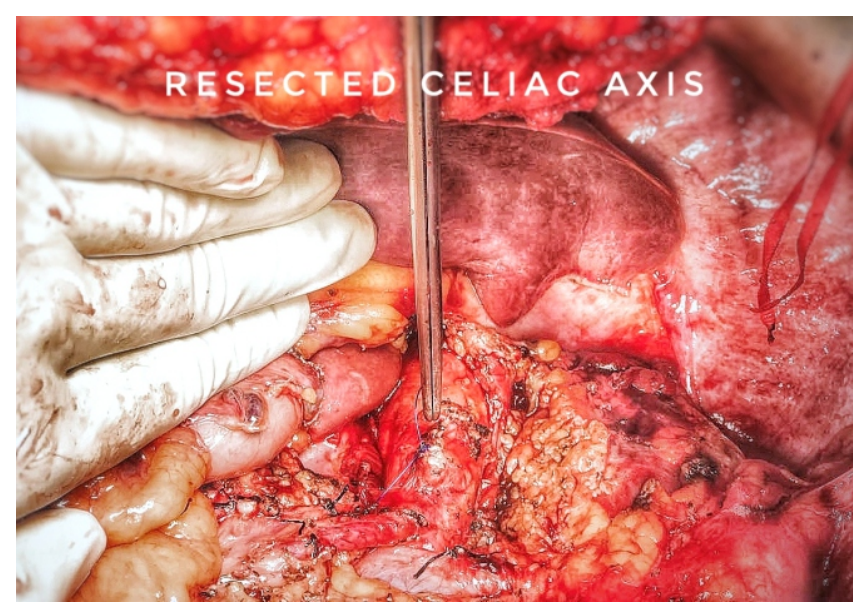

Figure 1. Post DP-CAR

aorta. Aorta was cleared on the sides to place an aortic clamp. CA was excised after side clamping the aorta and the defect was sutured with 3.0 prolene. A distal pancreatectomy with splenectomy was performed. The posterior resection margin was cleared to achieve complete exposure of the left renal vein. The proximal pancreatic margin was closed with 5.0 prolene. The drain was kept at the pancreatic transection site.

The patient had an uneventful recovery. On day 0 serum lactate was recorded highest $3.5(\mathrm{mmol} / \mathrm{L})$ and by day 1 it was reduced to $1.5(\mathrm{mmol} / \mathrm{L})$ while the recorded highest AST was on Day 2 (192U/L). An ultrasound scan was done in 2 weeks reconfirmed retrograde hepatic perfusion without any collections in the pancreatic bed.

Histology confirmed a moderately differentiated ductal adenocarcinoma on a background of chronic pancreatitis with negative resection margins at the root of CA and distal CHA. There were perineural invasion and infiltration of coeliac ganglions by the tumour. Four lymph nodes (peripancreatic) out of eighteen (Peripancreatic 7, perisplenic 11) were positive for tumour deposits. Tumour staging was pT4N2. The patient was referred for adjuvant chemotherapy.

Correspondence: Prabath Kumarasinghe

E-mail: ranganaksinghe14@gmail.com

(i) https://orcid.org/0000-0002-5078-0944

Received: 26-02-2021 Accepted: 20-03-2021

DOI: http://doi.org/10.4038/sljs.v39i1.8796

The Sri Lanka Journal of Surgery 2021; 39(1): 63-64 


\section{Discussion}

Appleby procedure was performed initially in 1953 for a locally advanced gastric cancer [2]. Since then it gained popularity and has been adopted to treat locally advanced pancreatic cancer. Careful patient selection is essential to delineate patients who may benefit from this extensive procedure. A crucial aspect of this procedure is to re-establish the blood supply to the liver after coeliac axis resection. There is a constantly existent pancreatoduodenal vascular arcade from the Superior mesenteric artery (SMA) connecting to the Gastroduodenal artery (GDA). If this arcade is patent CA resection can be done without vascular reconstruction.

Detailed imaging including MDCT with CA and SMA angiography is essential to delineate the local extent of the lesion and to assess the collaterals. The tumour must be confined to the pancreatic neck and body and the pancreatic head should be spared. Extension of the tumour must be confined to CA without the involvement of the aorta. Gastroduodenal artery, proper hepatic artery or SMA should not be involved by the tumour.

DP-CAR has mortality around 3\% even though it has high morbidity $(49 \%)[3,4]$. Common postoperative complications are pancreatic fistula, delayed gastric emptying and chylous ascites. Hepatic infarction is the most dreaded complication after DP-CAR.

In carefully selected patients DP-CAR offers a survival benefit over unresected locally advanced pancreatic ductal adenocarcinoma involving the coeliac axis [5]. Median overall survival after DP-CAR is $15-17$ months and 1-year survival is $60-87 \%$.

\section{Conclusion}

DP CAR is a complex surgical procedure for patients with locally advanced pancreatic neck and body carcinoma involving the coeliac axis. Favourable outcomes can be achieved with careful selection of patients before the procedure. A specially detailed evaluation of vascular anatomy and collateral circulation is essential to overcome serious complications after this procedure.

All authors disclose no conflict of interest. The study was conducted in accordance with the ethical standards of the relevant institutional or national ethics committee and the Helsinki Declaration of 1975, as revised in 2000 .

\section{References}

1. Pancreatic Adenocarcinoma Version 1.2020 NCCN Clinical Practice Guidelines in Oncology (NCCN Guidelines $\left.{ }^{\circledR}\right)$. Fort Washington, PA: National Comprehensive Cancer Network,Inc., 2020

2. APPLEBY LH. The coeliac axis in the expansion of the operation for gastric carcinoma. Cancer. $1953 \mathrm{Jul} ; 6(4): 704-7$. doi: $10.1002 / 1097-0142$ (195307)6:4<704::aid-cncr282 0060410>3.0.co;2-p. PMID: 13059764.

3. Gong H, Ma R, Gong J, Cai C, Song Z, Xu B. Distal Pancreatectomy With En Bloc Celiac Axis Resection for Locally Advanced Pancreatic Cancer: A Systematic Review and MetaAnalysis. Medicine (Baltimore). 2016 Mar;95(10):e3061. doi: 10.1097/MD.0000000000003061.

4. Zhou YM, Zhang XF, Li XD, Liu XB, Wu LP, Li B. Distal pancreatectomy with en bloc celiac axis resection for pancreatic body-tail cancer: Is it justified? Med Sci Monit. 2014 Jan 2;20:15. doi: 10.12659/MSM.889847. PMID: 24382572

5. Yamamoto Y, Sakamoto Y, Ban D, Shimada K, Esaki M, Nara S, Kosuge $\mathrm{T}$. Is celiac axis resection justified for T4 pancreatic body cancer? Surgery. 2012 Jan;151(1):61-9. doi: 10.1016/j.surg.2011.06.030. Epub 2011 Nov 14.

\section{Learning Points:}

- Surgical resection with curative intent is possible in locally advanced carcinoma of the pancreas.

- Detailed radiological assessment with coeliac axis and superior mesenteric artery angiography is essential in planning DPCAR.

- Common complications of DP-CAR includes pancreatic fistula, gastric ischemia and rarely hepatic necrosis. 